\title{
Reactive Oxygen Species Modulator 1 (Romo1) Predicts Poor Outcomes in Advanced Non-small Cell Lung Cancer Patients Treated with Platinum-Based Chemotherapy
}

\author{
Seung Hyeun Lee, MD, PhD \\ Sue In Choi, MD2 \\ Ji Sung Lee, $\mathrm{PhD}^{3}$ \\ Chul Hwan Kim, MD, PhD ${ }^{4}$ \\ Won Jai Jung, $M D^{2}$ \\ Eun Joo Lee, MD, PhD² \\ Kyung Hoon Min, MD, PhD² \\ Gyu Young Hur, MD, PhD² \\ Seung Heon Lee, MD, $\mathrm{PhD}^{2}$ \\ Sung Yong Lee, MD, $\mathrm{PhD}^{2}$ \\ Je Hyeong Kim, MD, PhD² \\ Sang Yeub Lee, MD, PhD² \\ Chol Shin, MD, PhD² \\ Jae Jeong Shim, MD, $P h D^{2}$ \\ Kyung Ho Kang, MD, PhD² \\ Kwang $\mathrm{Ho}$ In, MD, PhD²
}

\begin{abstract}
${ }^{1}$ Department of Internal Medicine, Kyung Hee University School of Medicine, Seoul, ${ }^{2}$ Division of Respiratory and Critical Care Medicine, Department of Internal Medicine, Korea University College of Medicine, Seoul, ${ }^{3}$ Clinical Research Center, Asan Medical Center, Seoul,

${ }^{4}$ Department of Pathology, Korea University College of Medicine, Seoul, Korea
\end{abstract}

Correspondence: Kwang Ho In, MD, PhD Division of Respiratory and Critical Care Medicine, Department of Internal Medicine, Korea University College of Medicine,

73 Inchon-ro, Seongbuk-gu, Seoul 02841, Korea Tel: 82-2-920-5316

Fax: 82-2-929-2045

E-mail: khin@kumc.or.kr

\section{Received March 28, 2016}

Accepted May 10, 2016

Published Online May 18, 2016

*Seung Hyeun Lee and Sue In Choi contributed equally to this work.

\section{Purpose}

Reactive oxygen species modulator 1 (Romo1) is a key mediator of intracellular reactive oxygen species production. However, examination of the clinical usefulness of Romo1 in cancers has been limited. We evaluated the association of Romo1 expression with clinical outcomes in advanced non-small cell lung cancer (NSCLC) patients treated with platinumbased chemotherapy.

\section{Materials and Methods}

Romo1 expression in tumor tissue was examined by immunohistochemistry and evaluated by histological score. Survival analyses were performed according to Romo1 expression and the association between Romo1 expression and clinical parameters was evaluated.

\section{Results}

A total of 88 tumor specimens were analyzed. Significantly shorter median progression-free survival (PFS) was observed in the high Romo1 group compared with the low Romo1 group (4.5 months vs. 9.8 months, $p<0.001$ ), and the median overall survival (OS) of the high Romo1 group was also significantly shorter than that of the low Romo1 group (8.4 months vs. 15.5 months, $p<0.001)$. Results of multivariate analyses showed significant association of high Romo1 expression with both poor PFS (hazard ratio [HR], 2.75; 95\% confidence interval [Cl], 1.71 to 4.44) and poor OS (HR, 3.99; 95\% Cl, 2.36 to 6.74). Results of the subgroup analysis showed a similar association regardless of tumor histology. Romo1 expression showed no association with any clinical parameter including age, sex, smoking status, stage, differentiation, or tumor histology.

\section{Conclusion}

Romo1 overexpression was associated with poor response to treatment and shorter survival in advanced NSCLC patients treated with platinum-based chemotherapy. Romo1 could be a potential adverse predictive marker in this setting.

Key words

Lung neoplasms, Biomarkers, Reactive oxygen species, Prognosis, Drug therapy, Platinum 


\section{Introduction}

With increasing incidence and mortality, lung cancer has become the leading cause of cancer-related death and a challenging clinical problem worldwide [1]. Non-small cell lung cancer is a major type of lung cancer accounting for $80 \%$ of lung cancer cases. Despite the development of early diagnostic modalities and tumor markers, it is often diagnosed at an advanced stage and the 5-year survival rate is less than $20 \%$ [2]. Despite increasing use of targeted agents including epidermal growth factor receptor (EGFR) tyrosine kinase inhibitor and anaplastic lymphoma kinase (ALK) inhibitor for treatment of lung cancer, platinum-based combination chemotherapy is still the standard first-line treatment for non-small cell lung cancer (NSCLC), particularly for tumors without EGFR mutation or ALK translocation [3].

The discovery and clinical application of biomarkers is critical not only for early detection of the disease but also for the identification of patients expected to show the best response to therapy. Despite numerous studies, there is no biomarker with clinical significance for the prediction of outcomes after platinum-based chemotherapy. The KRAS mutation, despite its association with poor survival of NSCLC patients in several studies [4,5], does not appear to be predictive of chemotherapeutic efficacy [6,7]. Excision repair cross-complementation group 1 (ERCC1) has been linked with resistance to platinum-based chemotherapy in NSCLC [8,9]. Low ERCC1 levels are related to increased recurrence in untreated patients after lung resection and correlated with prolonged survival of NSCLC patients treated with platinum-based adjuvant and palliative chemotherapy [10-12]. However, ERCC1-tailored chemotherapy failed to prove its utility in a prospective randomized trial [13]. Thus, discovery of novel biomarkers is needed to improve therapeutic outcomes.

Reactive oxygen species modulator 1 (Romo1) is a novel protein first cloned from head and neck cancer tissue in 2006. It is located in the mitochondrial membrane and is a key modulator of intracellular reactive oxygen species [14]. Upregulation of Romo1 was reported in a variety of cancer cells and Romo1-induced reactive oxygen species (ROS) production is essential for the proliferation of both normal and cancer cells [15]. Interestingly, Romo1-derived ROS production is related to chemoresistance in lung cancer cells [16]. In a recent study, we demonstrated that Romo1 expression is increased in lung tumor tissue compared to normal surrounding tissue [17]. In addition, we found that serum Romo1 levels are significantly increased in lung cancer patients as compared to a cancer-free population [17], and that Romo1 overexpression was associated with poor clinical outcome in NSCLC patients who underwent surgical resection [18]. However, few studies regarding the clinical impli- cations of Romo1 in advanced NSCLC have been reported.

The aim of this study is to examine the question of whether Romo1 protein expression is associated with responses to treatment and survival rates in NSCLC patients who received platinum-based chemotherapy.

\section{Materials and Methods}

\section{Patients and data collection}

Patients with advanced NSCLC who received platinumbased doublet as first-line chemotherapy at Korea University Anam Hospital from May 2008 to March 2010 were recruited retrospectively. Formalin-fixed paraffin-embedded tumor specimens acquired by percutaneous needle biopsy or endoscopic bronchial biopsy were used for evaluation of Romo1 expression. Patients who died within 1 month after diagnosis, those with a history of other cancers, and those who had received chemotherapy or radiation therapy were excluded. During the study period, of 123 patients who underwent first-line chemotherapy with platinum-based doublet for NSCLC, tissue specimens were available from 102 patients. Six cases were excluded because the samples were unsuitable for immunohistochemical staining, five cases were excluded due to unavailable survival data, and three cases were excluded according to the exclusion criteria. Thus, a total of 88 patients were enrolled.

All patients underwent chest computed tomography, brain magnetic resonance imaging, and ${ }^{18} \mathrm{~F}$-fluorodeoxyglucose positron emission tomography for clinical staging determined according to the International Association for the Study of Lung Cancer TNM staging classification of NSCLC [13]. Clinical data were reviewed retrospectively from the medical records. Tumor response was examined by computed tomography every two cycles and evaluated according to the Response Evaluation Criteria in Solid Tumors (RECIST) 1.1 as complete response (CR), partial response (PR), stable disease (SD), or progressive disease (PD) [19]. The study protocol was approved by the clinical research ethics committee of Korea University Anam Hospital.

\section{Quantification of Romo1 protein expression}

Romo1 protein expression was measured by immunohistochemical staining. Four-micron-thick sections were prepared for each specimen and the staining was performed using a BOND-MAX Immunoautostainer (Leica Biosystems, Newcastle Upon Tyne, UK). Slides were heated at $98^{\circ} \mathrm{C}$ for 20 minutes and cooled for 10 minutes in Epitope Retrieval 
Table 1. Clinical characteristics of patients according to low and high Romo1 expression

\begin{tabular}{|c|c|c|c|c|}
\hline \multirow{2}{*}{ Variable } & \multirow{2}{*}{ No. of patients (\%) } & \multicolumn{2}{|c|}{ Romo1 expression } & \multirow{2}{*}{ p-value } \\
\hline & & Low $(<200)$ & High $(\geq 200)$ & \\
\hline Total & $88(100)$ & $53(60)$ & $35(40)$ & \\
\hline \multicolumn{5}{|l|}{ Sex } \\
\hline Female & $23(26)$ & $13(25)$ & $10(29)$ & 0.672 \\
\hline Male & $65(74)$ & $40(75)$ & $25(71)$ & \\
\hline \multicolumn{5}{|l|}{ Age (yr) } \\
\hline$<65$ & $31(35)$ & $22(42)$ & $9(26)$ & 0.129 \\
\hline$\geq 65$ & $57(65)$ & $31(58)$ & $26(74)$ & \\
\hline \multicolumn{5}{|l|}{ Smoking } \\
\hline Never & $25(28)$ & $15(28)$ & $10(29)$ & 0.978 \\
\hline Ever & $63(72)$ & $38(72)$ & $25(71)$ & \\
\hline \multicolumn{5}{|l|}{ ECOG PS } \\
\hline 0,1 & $59(67)$ & $35(66)$ & $24(69)$ & 0.804 \\
\hline$\geq 2$ & $29(33)$ & $18(34)$ & $11(31)$ & \\
\hline \multicolumn{5}{|l|}{ T stage } \\
\hline $\mathrm{T} 1$ & $7(8)$ & $3(6)$ & $1(3)$ & 0.645 \\
\hline$\geq \mathrm{T} 2$ & $81(92)$ & $49(94)$ & $34(97)$ & \\
\hline \multicolumn{5}{|l|}{ N stage } \\
\hline No & $9(10)$ & $5(10)$ & $3(9)$ & $>0.99$ \\
\hline$\geq \mathrm{N} 1$ & $79(90)$ & $47(90)$ & $32(91)$ & \\
\hline \multicolumn{5}{|l|}{ Stage } \\
\hline IIIB & $25(28)$ & $16(30)$ & $9(26)$ & 0.648 \\
\hline IV & $63(72)$ & $37(70)$ & $26(74)$ & \\
\hline \multicolumn{5}{|l|}{ EGFR mutation ${ }^{\text {a) }}$} \\
\hline Positive & $6(37)$ & $3(33)$ & $3(43)$ & 0.781 \\
\hline Negative & $10(63)$ & $6(67)$ & $4(57)$ & \\
\hline \multicolumn{5}{|l|}{ Differentiation } \\
\hline Well & $61(69)$ & $39(74)$ & $22(63)$ & 0.285 \\
\hline Mod-poor & $27(31)$ & $14(26)$ & $13(37)$ & \\
\hline \multicolumn{5}{|l|}{ Tumor histology } \\
\hline $\mathrm{ADC}$ & $50(57)$ & $32(60)$ & $18(51)$ & 0.171 \\
\hline SQCC & $38(43)$ & $23(40)$ & $15(49)$ & \\
\hline \multicolumn{5}{|l|}{ First-line treatment } \\
\hline Pemetrexed/Platinum & $30(34)$ & $16(53)$ & $14(47)$ & 0.948 \\
\hline Gemcitabine/Platinum & $36(40)$ & $20(56)$ & $16(44)$ & \\
\hline Paclitaxel/Platinum & $14(16)$ & $8(57)$ & $6(43)$ & \\
\hline Docetaxel/Platinum & $8(9)$ & $4(50)$ & $4(50)$ & \\
\hline
\end{tabular}

Romo1, reactive oxygen species modulator 1; ECOG PS, Eastern Cooperative Oncology Group performance status; T, tumor; $\mathrm{N}$, lymph node; EGFR, epidermal growth factor receptor; ADC, adenocarcinoma; SQCC, squamous cell carcinoma. ${ }^{\text {a) } E G F R ~}$ mutation data were available in 16 adenocarcinoma patients.

Solution 1 and $0.01 \mathrm{M}$ citrate buffer ( $\mathrm{pH} 6.0$ ), respectively. Slides were then washed in distilled water, followed by blocking of endogenous peroxidase activity using a Bond Polymer Refine Detection Kit (Leica Biosystems) for 5 minutes. Slides were washed and placed in Tris-buffered saline, followed by incubation for 30 minutes with a Romo1 monoclonal antibody (OriGene Technologies, Rockville, MD) at a
1:200 dilution. Sections were developed with 3,3'-diaminobenzidine chromogen solution for 7 minutes, counterstained with hematoxylin, and dehydrated. Human colon adenocarcinoma tissue was used as a positive control, and slides stained without the primary antibody were used as a negative control.

Histopathological assessment was performed separately 
A

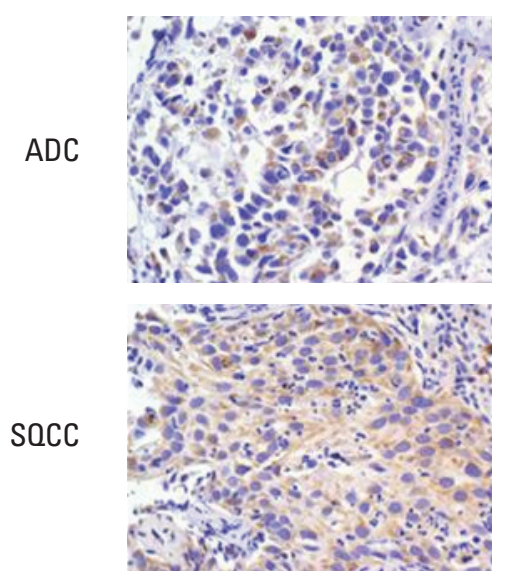

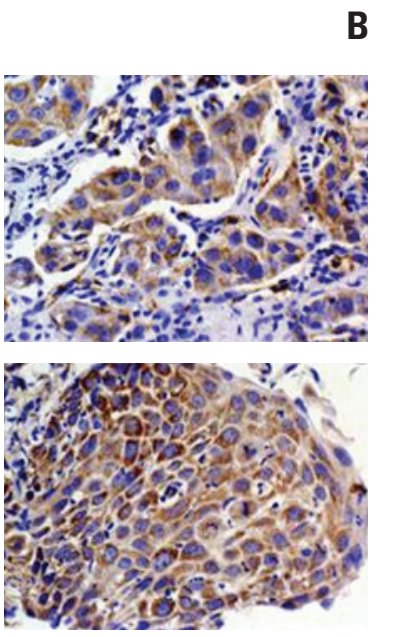

B

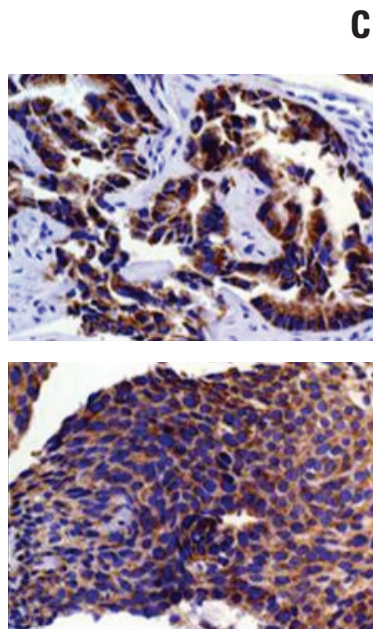

Fig. 1. Representative samples of immunochemical staining for Romo1 with different $\mathrm{H}$ scores ( $\times 200)$. Romo1 was primarily detected in the cytoplasm of adenocarcinoma and squamous cell carcinoma cells. (A) H score of 50. (B) H score of 150. (C) $\mathrm{H}$ score of 250. Romo1, reactive oxygen species modulator 1; H scores, histological scores; ADC, adenocarcinoma; SQCC, squamous cell carcinoma.

by two investigators (S.H.L. and S.I.C.) and then a consensus was made on discordant assessment. Stained cells were interpreted as positive when cytoplasmic staining was identified. Staining intensities of individual cells were graded as 0 (no staining), 1 (weak), 2 (distinct), or 3 (strong), and the percentages of cells with these staining intensities were calculated. Finally, histological scores (H scores) were calculated by multiplying staining intensities by the percentages of cells with each staining intensity (possible range, 0 to 300 ).

\section{Statistical analyses}

The cutoff $\mathrm{H}$ score for discriminating between low and high Romo1 expression was defined as the point with the lowest $\mathrm{p}$-value on the log-rank test for all possible $\mathrm{H}$ scores. Clinical outcomes were assessed using response rate (RR), progression-free survival (PFS), and overall survival (OS). $R R$ was defined as the percentage of patients with $C R$ and PR among the population. PFS was defined as the time from the first day of chemotherapy to disease progression or death from any cause. OS was defined as the time from the first day of chemotherapy to death from any cause. Chi-square test or Fisher exact test was used as appropriate for analysis of the proportions of low and high $\mathrm{H}$ scores in the different patient groups. Associations between clinical parameters and survival were first evaluated by univariate analysis using the log-rank test, and multivariate Cox's proportional hazard regression was performed with adjustment for parameters with p-values of less than 0.3 in the univariate analysis. Sur- vival curves were generated using the Kaplan-Meier method. p-values of less than 0.05 were considered significant. Statistical analyses were performed using SPSS ver. 19.0 for Windows (SPSS Inc., Chicago, IL).

\section{Results}

\section{Patients' characteristics}

The clinical characteristics of the study population are summarized in Table 1. All subjects were Korean and the median age was 69 years (range, 39 to 81 years). Sixty-five patients $(74 \%)$ were male, and 57 patients $(65 \%)$ were over 65 years of age. Sixty-three patients $(72 \%)$ were current or former smokers. Fifty-nine patients $(67 \%)$ had an Eastern Cooperative Oncology Group (ECOG) performance status of 0 or 1 , and 25 patients (28\%) were stage IIIB and 63 patients $(72 \%)$ were stage IV. Twenty-five patients $(28 \%)$ had welldifferentiated cancer and 63 patients $(72 \%)$ had moderatelyto poorly-differentiated cancer. Fifty patients $(57 \%)$ had adenocarcinoma and 38 patients $(43 \%)$ had squamous cell carcinoma. EGFR mutation analysis was performed in only 16 adenocarcinoma patients; six patients $(37 \%)$ were positive for the activating mutation while 10 patients $(63 \%)$ were negative.

The chemotherapy regimens used for first-line treatment 


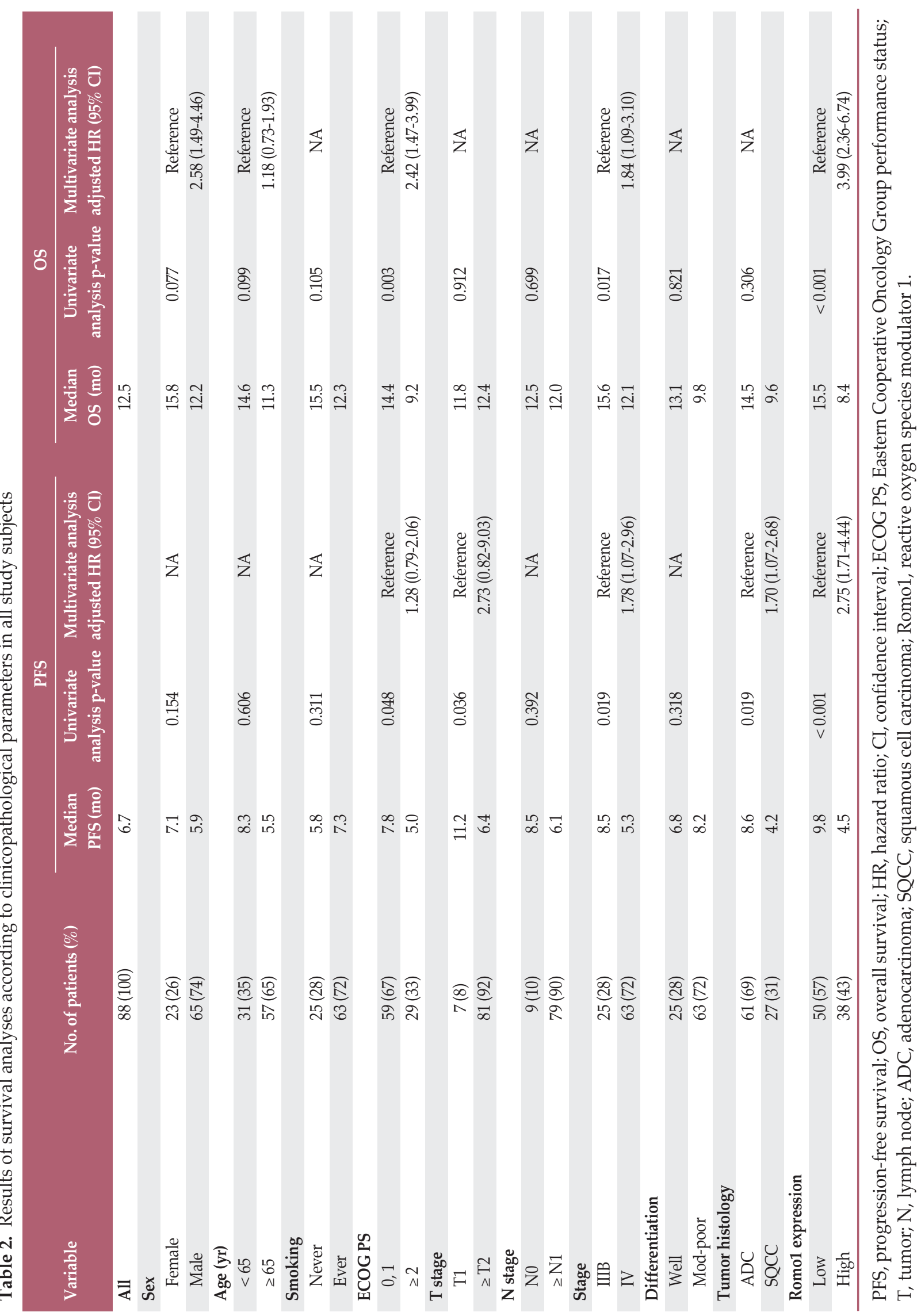


were pemetrexed / platinum ( $\mathrm{n}=30,34 \%)$, gemcitabine/platinum $(\mathrm{n}=36,40 \%)$, paclitaxel/platinum $(\mathrm{n}=14,16 \%)$, and docetaxel/ platinum $(n=8,9 \%)$. The median number of cycles of first-line chemotherapy was 4 (range, 1 to 9). Of the 88 patients, 48 patients ( $54.0 \%$ ) underwent second-line or later treatment during the follow-up period. Second-line treatment regimens were gemcitabine/vinorelbine $(n=11,23 \%)$, pemetrexed $(n=14,29 \%)$, docetaxel $(n=4,8 \%)$, erlotinib $(n=10$, $21 \%)$, and gefitinib $(n=9,20 \%)$. Twenty-one patients underwent third-line treatment with gemcitabine/vinorelbine $(n=8,38 \%)$, pemetrexed $(n=3,14 \%)$, docetaxel $(n=2,9 \%)$, or gefitinib $(n=8,38 \%)$.

\section{Romo1 protein expression}

Representative slides with different $\mathrm{H}$ scores are shown in Fig. 1. Romo1 protein was primarily localized to the cytoplasm of cancer cells. The Romo1 H scores were normally distributed with a median of 180.7 (range, 17 to 295).

\section{Relationship between Romo1 expression and clinical parameters}

The optimal cutoff $\mathrm{H}$ score determined for discriminating between low and high Romo1 expression was 200. Using this cutoff, 53 patients (60\%) were allocated to the low Romo1 group and 35 patients (40\%) to the high Romo1 group.

To determine which clinical parameters are associated with the level of Romo1 expression, we compared the proportion of low and high Romo1 expression in the different patient groups. There was no difference in the proportion of patients with low and high Romo1 expression in all patients groups (Table 1). This result indicates that none of the clinical parameters including age, sex, smoking status, performance status, stage, tumor differentiation, or histological type were associated with Romo1 expression.

\section{Relationship between Romo1 protein expression and RR}

Forty-one patients $(46 \%)$ showed PR, 11 patients $(12 \%)$ showed SD, and 36 patients (42\%) showed PD. Thus, the overall RR was $46 \%$. In patients with low Romo1 expression, 29 patients (55\%) showed PR while 12 patients $(36 \%)$ in the high Romo1 group had PR. Significantly higher RR was observed in the low Romo1 group compared with that in the high Romo1 group ( $\mathrm{p}=0.017$ ). Clinical parameters including female sex, young age, never smoker, well-differentiated cancer, and better performance status showed a trend toward a good response to treatment but were not statistically significant.

\section{Relationship between Romo1 expression and PFS}

Results of survival analyses according to the clinical parameters are summarized in Table 2. The median PFS for the whole population was 6.7 months $(95 \%$ confidence interval [CI], 2.2 to 10.8). In the univariate analysis, poor performance, advanced $\mathrm{T}$ stage, advanced stage, and squamous histology showed association with poor PFS, and high Romo1 expression showed association with poor PFS (4.5 months vs. 9.8 months, $\mathrm{p}<0.001)$. In the multivariate analysis, advanced stage (hazard ratio [HR], 1.78; $95 \% \mathrm{CI}, 1.07$ to

A
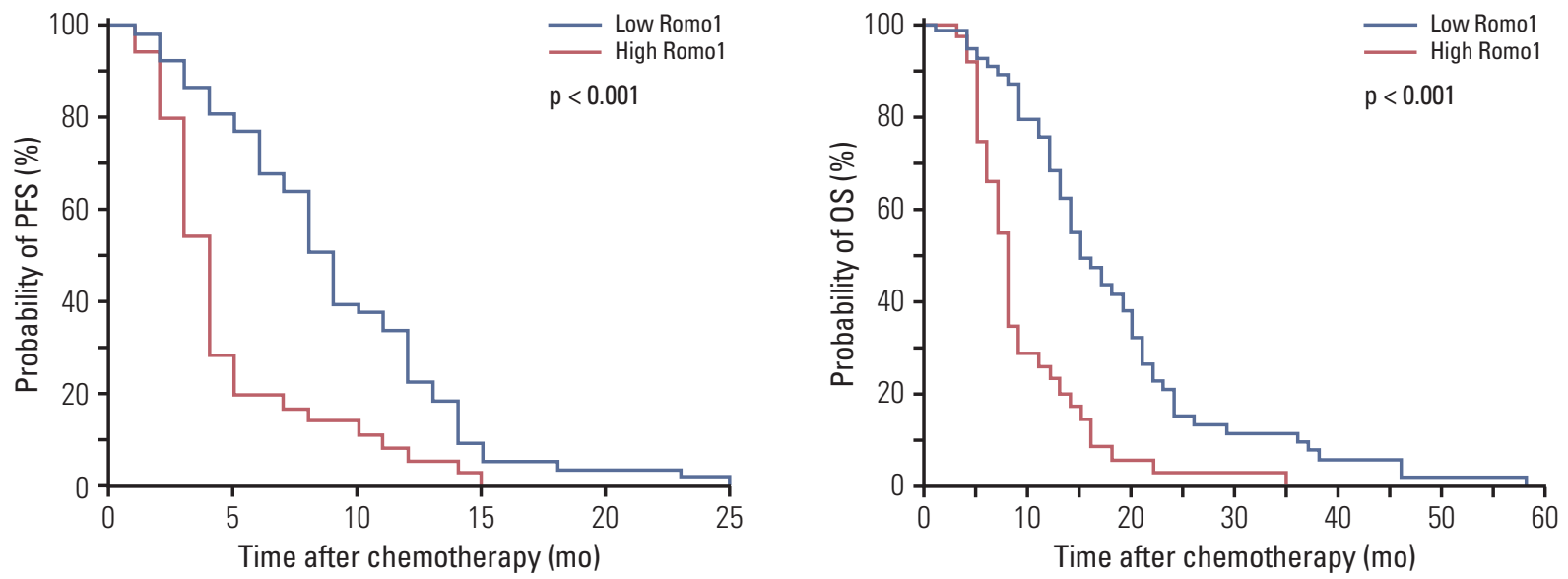

Fig. 2. Kaplan-Meier survival curves for progression-free survival (PFS) (A) and overall survival (OS) (B) in the overall population. p-values were determined using the log-rank test. Romo1, reactive oxygen species modulator 1. 
2.96) and high Romo1 expression (HR, 2.75; 95\% CI, 1.71 to 4.44) showed significant association with poor PFS. KaplanMeier survival curves showed that poor survival was likely in terms of PFS for patients with high Romo1 expression (Fig. 2A).

\section{Relationship between Romo1 expression and OS}

The median OS for all study subjects was 12.5 months $(95 \%$ CI, 9.8 to 15.5). In univariate analysis, poor performance status and advanced stage showed significant association with poor OS, and high Romo1 expression also showed significant association with poor OS (15.5 months vs. 8.4 months, $\mathrm{p}<0.001)$. In multivariate analysis, male gender $(\mathrm{HR}, 2.58$; 95\% CI, 1.49 to 4.46 ), poor performance status (HR, 2.42; $95 \%$ CI, 1.47 to 3.99 ), advanced stage (HR, $1.84 ; 95 \% \mathrm{CI}, 1.09$ to 3.10), and high Romo1 expression (HR, 3.99; 95\% CI, 2.36 to 6.74) showed significant association with poor OS. KaplanMeier survival curves showed that poor survival in terms of OS was likely in patients with high Romo1 expression (Fig. 2B).

\section{Subgroup analyses according to tumor histology}

Further survival analyses were performed according to tumor histology. The median PFS of patients with adenocarcinoma was 8.8 months (95\% CI, 4.6 to 12.5). In univariate analysis patients with adenocarcinoma, male gender, smoking history, advanced stage, and high Romol expression showed significant association with poor PFS and in multivariate analysis, high Romo1 expression showed association with poor PFS (HR, 2.43; 95\% CI, 1.42 to 3.67). In terms of OS, the median survival was 14.6 months (95\% CI, 11.7 to 17.4). In univariate analysis, old age, smoking history, poor performance, advanced stage, and high Romo1 expression showed significant association with poor OS. In multivariate analysis, poor performance (HR, 2.91; 95\% CI, 1.46 to 5.79), advanced stage (HR, 1.87; 95\% CI, 1.16 to 3.81), and high Romo1 expression (HR, 2.08; 95\% CI, 1.03 to 4.21) showed significant association with shorter OS.

In patients with squamous cell carcinoma, the median PFS was 4.5 months (95\% CI, 2.7 to 7.1). In univariate analysis high Romo1 expression showed association with poor PFS, and in the multivariate analysis, high Romo1 expression showed significant association with poor PFS (HR, 4.41; $95 \%$ CI, 2.94 to 8.69). In terms of OS, the median survival was 9.8 months (95\% CI, 6.8 to 12.4). In univariate analysis advanced stage and high Romo1 expression showed association with poor OS, and in the multivariate analysis, advanced stage (HR, 1.15; 95\% CI, 1.07 to 2.38) and high Romo1 expression (HR, 3.30; 95\% CI, 2.65 to 9.10) showed significant association with poor OS. The Kaplan-Meier survival curves showed that poor survival in terms of PFS and OS in both cell types was likely for patients with high Romo1 expression.

\section{Discussion}

In the current study, Romo1 overexpression was significantly associated with poor PFS and OS in advanced NSCLC patients who received platinum-based chemotherapy as first line treatment. These findings were consistent regardless of tumor histology. To the best of our knowledge, this is the first study demonstrating the relation of Romo1 expression to clinical outcome as well as its potential as a predictive marker in advanced NSCLC.

Although Romo1 was discovered a decade ago, the clinical significance of this protein is not well known. Several in vitro studies have suggested possible involvement of Romo1 in many steps in carcinogenesis, including tumor progression and invasion $[14,16,20,21]$. Based on our previous findings $[17,18]$, we hypothesized that Romo1 might have clinical implications in advanced lung cancer. The current study demonstrates the potential of Romo1 in prediction of clinical outcomes not only in early stage lung cancer but also in advanced disease. In addition, the consistently positive findings of Romo1 in lung cancers may provide a foundation for future studies examining the clinical usefulness of this protein in other malignancies.

Although the exact mechanism by which Romo1 overexpression is related to poor response and shorter survival of patients who received platinum-based treatment is unclear, adaptation to oxidative stress could be a possible explanation. Adaption involves regulation of the redox buffering system and upregulation of antioxidant enzymes required for survival of cancer cells from external stimulation or therapeutic drugs [22]. The anticancer activity of platinum is mainly the result of crosslinking with DNA and inhibition of DNA synthesis; however, induction and accumulation of ROS is another important mechanism involved in its activity [23]. Romo1 is a key mediator that maintains intracellular ROS production and is essential for survival in both normal and cancer cells $[15,24]$. Thus, we can hypothesize that Romo1-induced ROS production enhances expression of various antioxidants, resulting in chemoresistance to platinumrelated oxidative stress. Although further in vitro studies are required to confirm our hypothesis, it is supported by a previous finding that Romo1 is responsible for upregulation of antioxidant enzymes and the adaptive response to 5-fluorouracil treatment in lung cancer cells [16].

In the current study, we found no difference in expression levels of Romolbetween adenocarcinoma and squamous cell carcinoma, and the association between Romo1 expression 
and clinical outcome was similar regardless of tumor histology. This result is consistent with those of previous studies reporting Romo1 overexpression in NSCLC patients with both cell types $[17,18]$. Based on these findings, Romo1 overexpression can be considered as a common phenomenon and Romo1 has potential as a novel biomarker applicable to both cell types. However, considering the relatively closer relationship with cigarette smoking and the less frequent association of driving mutations in squamous cell carcinoma than in adenocarcinoma, further experiments are required to elucidate possible differences in the clinical implications of Romo1 between different cell types.

There are several limitations in this study. First, this study was conducted retrospectively at a single institution and included a relatively small sample size. Second, immunohistochemical staining was performed for the quantification of Romo1 protein expression, which may be less sensitive than other methods. However, a standard quantification method for Romo1 expression has not been established and immunohistochemistry is a widely used method for measurement of protein expression. Third, EGFR mutation data were available in only a small proportion of the subjects; therefore, analysis of the relationship between EGFR status and Romo1 expression was not possible. Most of our study subjects were enrolled before the IPASS trial [25]; since that trial EGFR mutation analysis has become an essential test for newlydiagnosed NSCLC patients.

\section{Conclusion}

In summary, Romo1 showed potential as an adverse predictive marker in NSCLC patients treated with platinumbased chemotherapy. Further large-scale investigations are required to determine the clinical implications of Romo1 in different clinical settings involving patients treated with targeted agents and using different quantification methods.

\section{Conflicts of Interest}

Conflict of interest relevant to this article was not reported.

\section{References}

1. Jemal A. Global burden of cancer: opportunities for prevention. Lancet. 2012;380:1797-9.

2. DeSantis CE, Lin CC, Mariotto AB, Siegel RL, Stein KD, Kramer JL, et al. Cancer treatment and survivorship statistics, 2014. CA Cancer J Clin. 2014;64:252-71.

3. Schiller JH, Harrington D, Belani CP, Langer C, Sandler A, Krook J, et al. Comparison of four chemotherapy regimens for advanced non-small-cell lung cancer. N Engl J Med. 2002;346: 92-8.

4. Slebos RJ, Kibbelaar RE, Dalesio O, Kooistra A, Stam J, Meijer $\mathrm{CJ}$, et al. K-ras oncogene activation as a prognostic marker in adenocarcinoma of the lung. N Engl J Med. 1990;323:561-5.

5. Tsao MS, Aviel-Ronen S, Ding K, Lau D, Liu N, Sakurada A, et al. Prognostic and predictive importance of p53 and RAS for adjuvant chemotherapy in non small-cell lung cancer. J Clin Oncol. 2007;25:5240-7.

6. Miller VA, Riely GJ, Zakowski MF, Li AR, Patel JD, Heelan RT, et al. Molecular characteristics of bronchioloalveolar carcinoma and adenocarcinoma, bronchioloalveolar carcinoma subtype, predict response to erlotinib. J Clin Oncol. 2008;26: 1472-8.

7. Roberts PJ, Stinchcombe TE. KRAS mutation: should we test for it, and does it matter? J Clin Oncol. 2013;31:1112-21.

8. Altaha R, Liang X, Yu JJ, Reed E. Excision repair cross complementing-group 1: gene expression and platinum resistance.
Int J Mol Med. 2004;14:959-70.

9. Rosell R, Lord RV, Taron M, Reguart N. DNA repair and cisplatin resistance in non-small-cell lung cancer. Lung Cancer. 2002;38:217-27.

10. Lord RV, Brabender J, Gandara D, Alberola V, Camps C, Domine $\mathrm{M}$, et al. Low ERCC1 expression correlates with prolonged survival after cisplatin plus gemcitabine chemotherapy in non-small cell lung cancer. Clin Cancer Res. 2002;8:2286-91.

11. Olaussen KA, Dunant A, Fouret P, Brambilla E, Andre F, Haddad V, et al. DNA repair by ERCC1 in non-small-cell lung cancer and cisplatin-based adjuvant chemotherapy. N Engl J Med. 2006;355:983-91.

12. Simon GR, Sharma S, Cantor A, Smith P, Bepler G. ERCC1 expression is a predictor of survival in resected patients with non-small cell lung cancer. Chest. 2005;127:978-83.

13. Cobo M, Isla D, Massuti B, Montes A, Sanchez JM, Provencio $\mathrm{M}$, et al. Customizing cisplatin based on quantitative excision repair cross-complementing 1 mRNA expression: a phase III trial in non-small-cell lung cancer. J Clin Oncol. 2007;25:274754.

14. Chung YM, Kim JS, Yoo YD. A novel protein, Romo1, induces ROS production in the mitochondria. Biochem Biophys Res Commun. 2006;347:649-55.

15. Na AR, Chung YM, Lee SB, Park SH, Lee MS, Yoo YD. A critical role for Romo1-derived ROS in cell proliferation. Biochem 
Biophys Res Commun. 2008;369:672-8.

16. Hwang IT, Chung YM, Kim JJ, Chung JS, Kim BS, Kim HJ, et al. Drug resistance to 5-FU linked to reactive oxygen species modulator 1. Biochem Biophys Res Commun. 2007;359:304-10.

17. Lee SH, Lee JS, Lee EJ, Min KH, Hur GY, Lee SH, et al. Serum reactive oxygen species modulator 1 (Romo1) as a potential diagnostic biomarker for non-small cell lung cancer. Lung Cancer. 2014;85:175-81.

18. Lee SH, Min JW, Lee JS, Kim CH, Yoo YD, Lee EJ, et al. Reactive oxygen species modulator 1 (Romo1) overexpression is an independent predictor of poor survival in NSCLC patients who undergo surgical resection. Lung Cancer. 2015;87:45-52.

19. Eisenhauer EA, Therasse P, Bogaerts J, Schwartz LH, Sargent D, Ford R, et al. New response evaluation criteria in solid tumours: revised RECIST guideline (version 1.1). Eur J Cancer. 2009;45:228-47.

20. Chung YM, Lee SB, Kim HJ, Park SH, Kim JJ, Chung JS, et al. Replicative senescence induced by Romo1-derived reactive oxygen species. J Biol Chem. 2008;283:33763-71.

21. Chung JS, Park S, Park SH, Park ER, Cha PH, Kim BY, et al. Overexpression of Romo1 promotes production of reactive oxygen species and invasiveness of hepatic tumor cells. Gastroenterology. 2012;143:1084-94.

22. Sosa V, Moline T, Somoza R, Paciucci R, Kondoh H, LLeonart ME. Oxidative stress and cancer: an overview. Ageing Res Rev. 2013;12:376-90.

23. Pelicano H, Carney D, Huang P. ROS stress in cancer cells and therapeutic implications. Drug Resist Updat. 2004;7:97-110.

24. Chung JS, Lee SB, Park SH, Kang ST, Na AR, Chang TS, et al. Mitochondrial reactive oxygen species originating from Romo1 exert an important role in normal cell cycle progression by regulating p27(Kip1) expression. Free Radic Res. 2009;43:729-37.

25. Mok TS, Wu YL, Thongprasert S, Yang CH, Chu DT, Saijo N, et al. Gefitinib or carboplatin-paclitaxel in pulmonary adenocarcinoma. N Engl J Med. 2009;361:947-57. 